\title{
Efecto de la congelación de leche caprina sobre la estabilidad oxidativa, calidad higiénico sanitaria y de composición en un rebaño de la raza Saanen
}

\section{Effect of goat milk freezing on oxidative stability, hygienic sanitary and composition quality in a Saanen breed herd}

\author{
Grille, Lucía $^{(1)}$, Carro, Silvana ${ }^{(1)}$, Escobar, Daniela ${ }^{(4)}$, Fros, Carolina ${ }^{(3)}$, Cousillas, Georgina ${ }^{(3)}$, Lazzarini, Florencia ${ }^{(3)}$, \\ Borges, Alejandra $^{(4)}$, González, Silvana ${ }^{(2)}$ \\ (1) Departamento de Ciencia y Tecnología de la Leche, Facultad de Veterinaria, UdelaR, Uruguay - (2) Parque de Ac- \\ tividades Agroindustriales, PAGRO, Unidad Montevideo Rural, Intendencia de Montevideo, Uruguay - ${ }^{(3)}$ Facultad \\ de Veterinaria, UdelaR (trabajo final de grado) - ${ }^{(4)}$ Gerencia de I+D+I, Laboratorio Tecnológico del Uruguay, LATU, \\ Uruguay.
}

Contacto: Igrille@gmail.com

Recibido: 29/5/2013 - Aprobado: 6/12/2013

\begin{abstract}
$\underline{\text { Resumen }}$
En establecimientos caprinos para la conservación de la leche es común aplicar la tecnología de congelación, ya sea para venta directa o para la elaboración de quesos. En este trabajo se estudió la congelación a $-18{ }^{\circ} \mathrm{C}$ como método de conservación de la leche caprina durante 6 meses. Se evaluó el efecto de la congelación en leche cruda y en leche pasteurizada. Se utilizaron dos métodos de descongelado: baño María $\left(27^{\circ} \mathrm{C}\right)$ y heladera $\left(4^{\circ} \mathrm{C}\right)$. En los días $0,60,120$ y 180 se analizó la composición (\% materia grasa, \% proteínas totales, \% lactosa, \% sólidos totales y \% sales), parámetros fisicoquímicos ( $\mathrm{pH}$, acidez y densidad), parámetros microbiológicos (recuento de mesófilos aerobios totales, coliformes totales, Staphylococcus coagulasa positiva), recuento de células somáticas y la estabilidad a la oxidación (según tiempos de inducción a la oxidación por Rancimat). Se concluye que no se afectó la calidad de la leche de acuerdo a los parámetros de este estudio durante la congelación por el período de 6 meses. Como método de conservación podría ser una alternativa para los establecimientos rurales. No se encontró diferencia en los métodos de descongelación evaluados.

Palabras clave: Conservación, leche caprina, congelación.
\end{abstract}

\begin{abstract}
$\underline{\text { Abstract }}$
In goat milk farm, in order to prevent milk from going off, it is common to apply the freezing technique, either to sell to public or for cheese making. We studied the freezing at $-18^{\circ} \mathrm{C}$ as a method of conservation of goat milk for 6 months. We evaluated the effect of freezing on raw and pasteurized milk. Two thawed methods were evaluated: water bath $\left(27^{\circ} \mathrm{C}\right)$ and refrigerator $\left(4^{\circ} \mathrm{C}\right)$. The composition was analyzed at $0,60,120$ and 180 days (\% fatty matter, $\%$ total protein, \% lactose, \% total solids and \% salt), physicochemical parameters ( $\mathrm{pH}$, acidity and density), microbiological parameters (total aerobic mesophilic count, total coliforms, Staphylococcus coagulase positive), somatic cell count and the oxidation stability (oxidation induction times, OIT, according to Rancimat). The quality of milk was not affected according to the parameters in this work during a six months freezing period. It could be an alternative as a conservation method for farms. We did not find any difference in the defrosting methods studied.

Keywords: Conservation, goat milk, freezing.
\end{abstract}

\section{Introducción}

En Uruguay, la mayoría de los productores de leche caprina posee tambos de pequeña extensión donde la mayor parte de la producción se destina a la elaboración de quesos $(70 \%)$ y el resto a consumo directo y alimentación de cabritos (Barberis, 2002). Estos productores se concentran en la zona suroeste del país (aproximadamente 16.000 $\mathrm{km}^{2}$ ), región que posee una larga tradición lechera. Generalmente, estos tambos de pequeña extensión emplean mano de obra familiar (Ciappesoni, 2006). En estos establecimientos se utiliza la tecnología de congelación, ya sea para venta directa o para materia prima, y así se hace posible mantener la elaboración de quesos artesanales en forma continua durante todo el año.

Para considerar una leche de calidad se deben tener en cuenta dos aspectos importantes: sus componentes y los factores contaminantes. La leche se puede afectar tanto en la propia glándula mamaria como en el medio ambiente, o incluso durante los procesos de la cadena de comercialización y procesamiento (Ávila, 2000).

La calidad higiénico-sanitaria puede ser evaluada por medio del recuento de células somáticas (RCS), que indica la frecuencia de animales con mastitis en el rebaño, y el recuento total de bacterias, que indica las condiciones de higiene y almacenamiento de la leche desde su obtención hasta el envío a la industria (Nunes de Sousa et al., 
2009). De acuerdo con Paape et al. (2007), el RCS se ha convertido en un parámetro aceptado para evaluar la calidad de leche y es la base de los programas de control mundiales de leche de vaca, oveja y cabra para prevenir el ingreso de la leche anormal o no apta para el consumo humano. Sin embargo, según Robertson y Muller (2005) en cabras es más difícil conectar el RCS con una posible infección intramamaria. Entre los microorganismos indicadores de calidad higiénico-sanitaria existentes, los más utilizados en leche son los coliformes totales y Staphylococcus coagulasa positiva. Cardoso (1985) plantea que los coliformes termotolerantes son indicadores de contaminación fecal y de riesgo de presencia de microorganismos patógenos que pueden causar toxiinfecciones en el consumidor. Los estafilococos son de gran importancia, principalmente los coagulasa positiva, pues son capaces de producir enterotoxinas termoestables que pueden llegar al consumidor después de la pasteurización.

La conservación de la leche es realizable, entre otros métodos, por medio de la refrigeración o incluso la congelación, que es uno de los métodos de conservación de la leche caprina que utilizan ciertos establecimientos con el fin de obtener mayor vida útil y mayor disponibilidad de la leche en distintas épocas del año.

Algunos autores hallaron que este método puede tener efectos adversos en la calidad, así como también en las propiedades fisicoquímicas, de composición y sensoriales (Needs, 1992). De la Fuente et al. (1997), Gomes et al. (1997) y Haenlein (2002) señalan que durante la congelación se puede dar una oxidación de la leche, de modo que se incrementa el contenido de ácidos grasos libres y, por lo tanto, la acidez de la leche, en especial si la lipasa no fue inactivada previamente por tratamiento térmico. Con respecto a la oxidación de lípidos, es una de las causas principales del deterioro de grasas y aceites y conduce a la pérdida de calidad (Meshref et al., 2008).

En cuanto a las proteínas, la congelación puede alterar su estructura en la leche y el queso (Fontecha et al., 1993).

De acuerdo a algunos autores, la congelación como método de conservación no altera las características microbiológicas de la leche y el producto, e inmediatamente después del descongelado tiene una calidad similar a la leche de la que se originó (Benedet y Schwinden, 1991; Gomes et al., 1997). Sin embargo, la congelación puede tener efectos negativos sobre las células bacterianas (Alur y Greez, 1975). Vilhena et al. (2008) exponen que la congelación de la leche puede alterar la pared celular de las bacterias perjudicando su capacidad de multiplicación, no obstante puede haber aumentos aparentes en los recuentos provocados por la separación de los aglomerados de bacterias durante el almacenamiento. Benedet y Schwinden (1991) y Gomes et al. (1997) demostraron que la congelación de la leche de cabra pasteurizada hasta por 90 días no altera significativamente sus características microbiológicas.

A pesar de que a nivel mundial no hay muchos estudios, algunos países como Brasil aceptan la aplicación de una temperatura igual o inferior a $-18{ }^{\circ} \mathrm{C}$, la cual debe alcanzarse en el menor tiempo posible (MAPA, 2000).

En Uruguay hasta el momento de la realización de este trabajo no existía recomendación oficial que contemplara la conservación de leche caprina a través de congelación. A partir de los resultados obtenidos, el Ministerio de Ganadería, Agricultura y Pesca, en su resolución $\mathrm{N}^{\circ} 27 / 011$, reglamenta la congelación de leche caprina a $-18{ }^{\circ} \mathrm{C}$ por el período de cinco meses (MGAP, 2011).

Este estudio se propone obtener resultados que avalen la conservación de leche caprina por medio de la congelación a $-18{ }^{\circ} \mathrm{C}$ durante seis meses, sin que se afecte la estabilidad oxidativa ni la calidad composicional y microbiológica. Para cumplir con lo anterior se definieron los siguientes objetivos específicos:

Evaluar y comparar, con respecto a la leche caprina cruda, cómo afecta el tratamiento térmico (pasteurización) previo a la congelación a los diferentes parámetros de calidad y estabilidad.

Evaluar el efecto de dos métodos de descongelado sobre los parámetros de calidad y de estabilidad de la leche caprina.

Evaluar cómo afecta el tiempo de congelación los valores referentes a calidad y estabilidad de leche caprina cruda y pasteurizada.

\section{Materiales y Métodos}

\section{Animales}

Se utilizaron 35 cabras de la raza Saanen, las cuales se encuentran en el Parque de Actividades Agropecuarias (PAGRO), perteneciente a la Intendencia de Montevideo, localizado en la zona de Colón, Montevideo, Uruguay.

\section{Diseño experimental}

Para cumplir con los objetivos planteados, se realizaron muestreos para análisis fisicoquímicos y microbiológicos según la metodología descripta por la Federación Internacional de Lechería (FIL-IDF 50C:1995)

Se realizaron 6 muestreos con un intervalo de 7 días cada uno, del total del volumen producido en el ordeñe matutino, en el momento en que los animales se encontraban en el pico de lactación. En cada muestreo y previa homogeneización se extrajeron 14 litros de leche del tanque, de los cuales 7 se envasaron como tal (leche cruda - LC), y los 7 restantes fueron sometidos a un proceso de pasteurización lenta LTLT ( $63^{\circ} \mathrm{C}$ durante 30 minutos) y se fraccionaron y envasaron como leche pasteurizada (LP). Luego del tratamiento térmico se utilizó la prueba de detección de la fosfatasa alcalina para verificar la efectividad del mismo. Antes de proceder a la congelación a $-18^{\circ} \mathrm{C}$ se extrajo una muestra de cada tipo de leche (LC y LP) y se le realizaron los análisis correspondientes al control de calidad. Estas muestras fueron consideradas como muestras control $(\mathrm{t} 0)$. Posteriormente se procedió a la congelación del resto de las muestras. La descongelación se llevó a cabo por medio de dos métodos: baño María (BM) y heladera $(\mathrm{H})$. La descongelación en $\mathrm{H}$ se realizó durante $60 \mathrm{hs} \mathrm{a} 4{ }^{\circ} \mathrm{C}$ y la de BM durante 2,5 hs a $27^{\circ} \mathrm{C}$. Estos tiempos fueron determinados previamente para asegurar la completa descongelación así como para no favorecer el crecimiento de microorganismos mesófilos durante el tiempo que duraba la descongelación por este último método. A los 60, 120 y 180 días de congelación ( $\mathrm{t} 1, \mathrm{t} 2$ y t3, respectivamente) se procedió a realizar la descongelación por los métodos anteriormente nombrados ( $\mathrm{H}$ y BM) de dos muestras de cada tipo de leche (LC y LP). Se obtuvieron las siguientes muestras: leche cruda baño María (LCBM), leche cruda heladera (LCH), leche pasteurizada baño María (LPBM) y leche pasteurizada heladera (LPH). Este ensayo se repitió 6 veces, constituyendo un total de 6 repeticiones (Tabla 1 ).

\begin{tabular}{|c|c|c|}
\hline Frecuencia de análisis & LC & LP \\
\hline t0: día 0 & $\begin{array}{c}\text { Previo a la congelación } \\
\text { y pasteurización }\end{array}$ & $\begin{array}{c}\text { Luego de pasteurizar y } \\
\text { previo a la congelación }\end{array}$ \\
\hline t1: 60 días & $\mathrm{BM}$ & $\mathrm{BM}$ \\
& $\mathrm{H}$ & $\mathrm{H}$ \\
\hline t2: 120 días & $\mathrm{BM}$ & $\mathrm{BM}$ \\
& $\mathrm{H}$ & $\mathrm{H}$ \\
\hline t3: 180 días & $\mathrm{BM}$ & $\mathrm{BM}$ \\
& $\mathrm{H}$ & $\mathrm{H}$ \\
\hline
\end{tabular}

Tabla 1. Muestreos de leche con los distintos tratamientos (LCBM, LCH, LPBM y LPH) y los diferentes tiempos (t0, t1, t2 y t3). 
Los recipientes en los que se envasó la leche para su posterior congelación fueron botellas cilíndricas, con capacidad de 1 litro, material: PET (Polietileno Tereftalato) con tapa rosca (de dimensiones estándar: $80 \mathrm{~mm}$ de diámetro x $230 \mathrm{~mm}$ de altura). Previo al envasado se sometió a cada uno de ellos a un proceso de limpieza y desinfección. Para este último procedimiento se utilizó ácido peracético $0,2 \%$ por 30 min asegurando que el ácido tomara contacto con toda la superficie del recipiente. Finalizado este tiempo se descartó el contenido de este ácido.

\section{Verificación de la pasteurización}

La comprobación de la efectividad del proceso térmico se comprobó a través de la determinación de la actividad de la fosfatasa alcalina (Silva et al., 1997), utilizando el kit comercial Phosphatesmo MI, $\mathrm{MN}{ }^{\circledR}$.

\section{Análisis de composición y fisicoquímicos}

Las muestras se analizaron en el laboratorio de calidad de leche del Departamento de Ciencia y Tecnología de la Leche de Facultad de Veterinaria (UdelaR) y en el Laboratorio Tecnológico del Uruguay (LATU). A cada muestra se le realizaron análisis de composición: materia grasa, proteína, lactosa, sólidos no grasos (SNG), sales (cenizas) y densidad, utilizando un equipo denominado Lactomilk ${ }^{\circledR}$ marca Lactoscan, modelo SLP, origen Bulgaria. Dicho equipo se calibró utilizando los métodos de referencia para cada análisis (materia grasa: Gerber; proteína: Kjeldahl; SNG: gravimétricos). El equipo analizador de leche presenta para cada componente analizado la siguiente precisión: materia grasa: $\pm 0,06 \%$; proteína: $\pm 0,15 \%$; lactosa: $\pm 0,2 \%$; SNG (sólidos no grasos): $\pm 0,15 \%$; sales (cenizas): \pm 0.05 ; densidad: $\pm 0,3 \mathrm{~kg} / \mathrm{m}^{3}$.

Se realizó recuento de células somáticas (RCS) mediante la técnica de Breed, FIL 148A (Internacional Dairy Federation, 1995) determinación de acidez Dörnic (Pinto et al., 1998) y pH mediante método de evaluación potenciométrico, utilizando un modelo de electrodos denominado Oakton ${ }^{\circledR}$.

\section{Análisis microbiológicos}

A cada muestra se le realizó análisis de recuento de mesófilos aerobios totales (RTMA), coliformes totales (CT) y Staphylococcus coagulasa positiva (ST) (APHA, 2001).

\section{Determinación de la estabilidad oxidativa}

Para evaluar la estabilidad oxidativa de la leche de cabra durante el almacenamiento se aplicó un método que utiliza condiciones de oxidación acelerada, temperaturas elevadas y presencia de oxígeno y aire en exceso, obteniendo resultados en períodos razonablemente cortos de tiempo.

El método de índice de estabilidad del aceite (OSI), también llamado Rancimat, permite medir la estabilidad oxidativa bajo condiciones estandarizadas (AOCS, 2009). El punto final es el correspondiente a un aumento repentino de ácidos volátiles generados a partir de las muestras de aceite calentado a alta temperatura bajo aireación constante. Estos compuestos se encuentran atrapados en agua y supervisados por electro-conductividad (Velasco et al., 2004).

Se midió la estabilidad oxidativa de la materia grasa de la leche. La extracción de la materia grasa se realizó según la norma ISO14156/ IDF172 (2001). Luego se determinaron los tiempos de inducción a la oxidación en el Rancimat (873), a $110^{\circ} \mathrm{C}$, flujo de aire $\mathrm{L} 7 \mathrm{~h}$, y toma de 2,5 g de muestra.

\section{Análisis estadísticos}

Las diferencias en los parámetros de calidad (composicional, fisicoquímicos y microbiológicos) obtenidas en los sucesivos muestreos para cada variable fueron analizadas estadísticamente mediante la prueba de análisis de varianza factorial en bloque, por parcelas divididas, con el programa INFOSTAT. Para determinar la diferencia entre los tiempos de congelado se realizó el Test de Tukey. Se consideran cambios significativos en cada variable cuando $\alpha$ sea $<0,05$. Los resultados microbiológicos y recuento de células somáticas (ufc/ml y cel $/ \mathrm{ml}$, respectivamente) se expresaron en log en base 10 para normalizar su distribución.

\section{Resultados}

Se evaluaron los parámetros de composición, fisicoquímicos y microbiológicos obtenidos al comparar los diferentes tratamientos: tratamiento térmico (LC y LP) y métodos de descongelado (BM y $\mathrm{H})$, donde se obtuvieron los siguientes resultados expresados en las Tablas 2,3 y 4 .

\begin{tabular}{|c|c|c|c|c|c|}
\hline & MG (\%) & $\begin{array}{c}\text { PROTEÍNA } \\
\text { (\%) }\end{array}$ & $\begin{array}{c}\text { LACTOSA } \\
\text { (\%) }\end{array}$ & SNG (\%) & Sales (\%) \\
\hline LP H & $3,56 \pm 0,66^{\mathrm{a}}$ & $2,69 \pm 0,08^{\mathrm{a}}$ & $3,90 \pm 0,11^{\mathrm{a}}$ & $7,36 \pm 0,20^{\mathrm{a}}$ & $0,68 \pm 0,01^{\mathrm{a}}$ \\
\hline LP BM & $3,61 \pm 0,67^{7^{\mathrm{b}}}$ & $2,70 \pm 0,08^{\mathrm{a}}$ & $3,91 \pm 0,12^{\mathrm{a}}$ & $7,38 \pm 0,18^{\mathrm{a}}$ & $0,68 \pm 0,01^{\mathrm{a}}$ \\
\hline LC H & $3,64 \pm 0,66^{\mathrm{ab}}$ & $2,69 \pm 0,08^{\mathrm{a}}$ & $3,90 \pm 0,11^{\mathrm{a}}$ & $7,36 \pm 0,20^{\mathrm{a}}$ & $0,68 \pm 0,01^{\mathrm{a}}$ \\
\hline LC BM & $3,65 \pm 0,57^{\mathrm{b}}$ & $2,69 \pm 0,08^{\mathrm{a}}$ & $3,89 \pm 0,13^{\mathrm{a}}$ & $7,36 \pm 0,11^{\mathrm{a}}$ & $0,67 \pm 0,11^{\mathrm{a}}$ \\
\hline
\end{tabular}

Tabla 2. Medias porcentuales de los valores de composición. Comparación entre los diferentes tratamientos (LPH, LPBM, LCH y LCBM) $(n=18)$.

*Medias con letra igual no muestran diferencia significativa $(p<0,05)^{\star} M G$ : materia grasa, SNG: sólidos no grasos.

\begin{tabular}{|c|c|c|c|}
\hline & $\mathbf{p H}$ & $\begin{array}{c}\text { Acidez } \\
\left({ }^{\mathbf{}} \mathbf{D}\right)\end{array}$ & $\begin{array}{c}\text { Densidad } \\
(\mathbf{g} / \mathbf{m l} \mathbf{l})\end{array}$ \\
\hline LP H & $6,63 \pm 0,10^{\mathrm{a}}$ & $14,25 \pm 0,20^{\mathrm{a}}$ & $1,028 \pm 0,01^{\mathrm{a}}$ \\
\hline LP BM & $6,63 \pm 0,08^{\mathrm{a}}$ & $14,21 \pm 0,22^{\mathrm{a}}$ & $1,028 \pm 0,01^{\mathrm{a}}$ \\
\hline LC H & $6,63 \pm 0,09^{\mathrm{a}}$ & $14,25 \pm 0,18^{\mathrm{a}}$ & $1,028 \pm 0,02^{\mathrm{a}}$ \\
\hline LC BM & $6,63 \pm 0,08^{\mathrm{a}}$ & $14,42 \pm 0,21^{\mathrm{a}}$ & $1,028 \pm 0,01^{\mathrm{a}}$ \\
\hline
\end{tabular}

Tabla 3. Valores medios de parámetros fisicoquímicos. Comparación entre los diferentes tratamientos (LPH, LPBM, LCH y LCBM) $(n=18)$. * Medias con letra igual no muestran diferencia significativa $(p<0,05)$.

\begin{tabular}{|c|c|c|c|c|}
\hline & $\begin{array}{c}\text { RMAT } \\
\text { (ufc/ml) }\end{array}$ & $\begin{array}{c}\text { CT } \\
\text { (ufc/ml) }\end{array}$ & $\begin{array}{c}\text { ST } \\
\text { (ufc/ml) }\end{array}$ & $\begin{array}{c}\text { RCS } \\
\text { (cel/ml) }\end{array}$ \\
\hline LP H & $0,62 \pm 0,89^{\mathrm{a}}$ & $0,13 \pm 0,80^{\mathrm{a}}$ & $0,00 \pm 0,00^{\mathrm{a}}$ & $6,24 \pm 0,30^{\mathrm{a}}$ \\
\hline LP BM & $0,85 \pm 0,40^{\mathrm{a}}$ & $0,07 \pm 0,75^{\mathrm{a}}$ & $0,00 \pm 0,00^{\mathrm{a}}$ & $6,24 \pm 0,28^{\mathrm{a}}$ \\
\hline LC H & $3,95 \pm 0,25^{\mathrm{b}}$ & $1,83 \pm 0,68^{\mathrm{b}}$ & $1,58 \pm 0,10^{\mathrm{b}}$ & $6,15 \pm 0,40^{\mathrm{a}}$ \\
\hline LC BM & $4,22 \pm 0,28^{\mathrm{b}}$ & $1,86 \pm 0,70^{\mathrm{b}}$ & $1,38 \pm 0,10^{\mathrm{b}}$ & $6,18 \pm 0,29^{\mathrm{a}}$ \\
\hline
\end{tabular}

Tabla 4. Valores medios en $\log _{10}$ para los parámetros microbiológicos y RCS, comparación entre los diferentes tratamientos (LPH, LPBM, LCH y LCBM) $(n=18)$.

* Medias con letra igual no muestran diferencia significativa $(p<0,05)$ * RMAT: recuento de mesófilos aerobios totales; CT: coliformes totales; ST: Staphylococcus coagulasa positiva; RCS: recuento de células somáticas. 
En las Tablas 5, 6 y 7 se observa la variación de los parámetros estudiados durante el tiempo de congelación (t0, t1, t2 y t3) para LC y LP.

\begin{tabular}{|c|c|c|c|c|c|c|c|c|c|c|}
\hline & \multicolumn{2}{|c|}{ MG \% } & \multicolumn{2}{|c|}{ Proteína \% } & \multicolumn{2}{|c|}{ Lactosa \% } & \multicolumn{2}{|c|}{ SNG 16\% } & \multicolumn{2}{|c|}{ Sales \% } \\
\hline & LC & LP & LC & LP & LC & LP & LC & LP & LC & LP \\
\hline to & $3,77 \pm 0,68^{b}$ & $3,79 \pm 0,60^{\mathrm{b}}$ & $2,74 \pm 0,08^{b}$ & $2,76 \pm 0,07^{\mathrm{c}}$ & $3,94 \pm 0,01^{\mathrm{a}}$ & $3,96 \pm 0,01^{\mathrm{b}}$ & $7,43 \pm 0,20^{\mathrm{a}}$ & $7,49 \pm 0,20^{\mathrm{b}}$ & $0,68 \pm 0,01^{a}$ & $0,69 \pm 0,01^{\mathrm{a}}$ \\
\hline t1 & $3,61 \pm 0,70^{\mathrm{a}}$ & $3,49 \pm 0,68^{\mathrm{a}}$ & $2,69 \pm 0,07^{\mathrm{ab}}$ & $2,72 \pm 0,08^{\mathrm{bc}}$ & $3,92 \pm 0,01^{\mathrm{a}}$ & $3,96 \pm 0,01^{\mathrm{b}}$ & $7,37 \pm 0,21^{\mathrm{a}}$ & $7,46 \pm 0,18^{b}$ & $0,68 \pm 0,01^{\mathrm{a}}$ & $0,68 \pm 0,01^{\mathrm{a}}$ \\
\hline t2 & $3,59 \pm 0,66^{\mathrm{a}}$ & $3,50 \pm 0,75^{\mathrm{a}}$ & $2,67 \pm 0,08^{\mathrm{a}}$ & $2,64 \pm 0,08^{\mathrm{a}}$ & $3,87 \pm 0,01^{\mathrm{a}}$ & $3,83 \pm 0,01^{\mathrm{a}}$ & $7,33 \pm 0,19^{\mathrm{a}}$ & $7,23 \pm 0,17^{\mathrm{a}}$ & $0,67 \pm 0,01^{\mathrm{a}}$ & $0,67 \pm 0,01^{\mathrm{a}}$ \\
\hline t3 & $3,63 \pm 0,72^{\mathrm{a}}$ & $3,56 \pm 0,76^{\mathrm{a}}$ & $2,67 \pm 0,07^{\mathrm{a}}$ & $2,67 \pm 0,08^{\mathrm{ab}}$ & $3,87 \pm 0,01^{\text {a }}$ & $3,87 \pm 0,01^{\mathrm{ab}}$ & $7,30 \pm 0,18^{\mathrm{a}}$ & $7,31 \pm 0,20^{\mathrm{ab}}$ & $0,67 \pm 0,01^{\mathrm{a}}$ & $0,67 \pm 0,01^{\mathrm{a}}$ \\
\hline
\end{tabular}

Tabla 5. Valores medios porcentuales de composición. Comparación entre los diferentes tiempos de congelación (t0, t1, t2 y t3) ( $\mathrm{n}=6$ ). *Medias con letra igual no muestran diferencia significativa $(p<0,05)$ *MG: materia grasa, SNG: sólidos no grasos.

\begin{tabular}{|c|c|c|c|c|c|c|}
\hline \multirow{2}{*}{} & \multicolumn{2}{|c|}{ pH } & \multicolumn{2}{c|}{ Acidez $\left({ }^{\circ} \mathbf{D}\right)$} & \multicolumn{2}{c|}{ Densidad (g/ml) } \\
\cline { 2 - 7 } & LC & LP & LC & LP & LC & LP \\
\hline t0 & $6,65 \pm 0,09^{\mathrm{b}}$ & $6,62 \pm 0,08^{\mathrm{b}}$ & $14,17 \pm 0,20^{\mathrm{a}}$ & $14,50 \pm 0,22^{\mathrm{c}}$ & $1,028 \pm 0,00^{\mathrm{a}}$ & $1,028 \pm 0,0^{\mathrm{a}}$ \\
\hline $\mathbf{t 1}$ & $6,64 \pm 0,15^{\mathrm{b}}$ & $6,66 \pm 0,12^{\mathrm{bc}}$ & $14,50 \pm 0,25^{\mathrm{a}}$ & $14,42 \pm 0,25^{\mathrm{a}}$ & $1,028 \pm 0,00^{\mathrm{a}}$ & $1,028 \pm 0,01^{\mathrm{a}}$ \\
\hline $\mathbf{t 2}$ & $6,70 \pm 0,10^{\mathrm{c}}$ & $6,71 \pm 0,10^{\mathrm{c}}$ & $14,25 \pm 0,12^{\mathrm{a}}$ & $13,92 \pm 0,19^{\mathrm{a}}$ & $1,028 \pm 0,00^{\mathrm{a}}$ & $1,028 \pm 0,0^{\mathrm{a}}$ \\
\hline $\mathbf{t 3}$ & $6,53 \pm 0,10^{\mathrm{a}}$ & $6,54 \pm 0,0^{\mathrm{a}}$ & $14,42 \pm 0,22^{\mathrm{a}}$ & $14,08 \pm 0,23^{\mathrm{a}}$ & $1,028 \pm 0,00^{\mathrm{a}}$ & $1,028 \pm 0,0^{\mathrm{a}}$ \\
\hline
\end{tabular}

Tabla 6. Valores medios de parámetros fisicoquímicos. Comparación entre los diferentes tiempos de congelación (t0, t1, t2 y t3) ( $\mathrm{n}=6)$.

* Medias con letra igual no muestran diferencia significativa $(p<0,05)$.

\begin{tabular}{|c|c|c|c|c|c|c|c|c|}
\hline \multirow{2}{*}{} & \multicolumn{2}{|c|}{$\begin{array}{c}\text { RMAT } \\
\text { (ufc/ml) }\end{array}$} & \multicolumn{2}{c|}{$\begin{array}{c}\text { CT } \\
\text { (ufc/ml) }\end{array}$} & \multicolumn{2}{|c|}{$\begin{array}{c}\text { St } \\
\text { (ufc/ml) }\end{array}$} & \multicolumn{2}{|c|}{$\begin{array}{c}\text { RCS } \\
\text { (cel/ml) }\end{array}$} \\
\cline { 2 - 9 } & $\mathbf{L C}$ & $\mathbf{L P}$ & $\mathbf{L C}$ & $\mathbf{L P}$ & $\mathbf{L C}$ & $\mathbf{L P}$ & $\mathbf{L C}$ & $\mathbf{L P}$ \\
\hline $\mathbf{t 0}$ & $4,78 \pm 0,78^{\mathrm{c}}$ & $0,43 \pm 0,22^{\mathrm{a}}$ & $3,71 \pm 0,55^{\mathrm{c}}$ & $0,10 \pm 0,19^{\mathrm{a}}$ & $1,51 \pm 0,15^{\mathrm{ab}}$ & $0,00 \pm 0,00^{\mathrm{a}}$ & $6,33 \pm 0,30^{\mathrm{b}}$ & $6,24 \pm 0,28^{\mathrm{a}}$ \\
\hline $\mathbf{t 1}$ & $4,15 \pm 0,67^{\mathrm{b}}$ & $0,69 \pm 0,20^{\mathrm{a}}$ & $2,04 \pm 0,48^{\mathrm{b}}$ & $0,08 \pm 0,15^{\mathrm{a}}$ & $1,53 \pm 0,12^{\mathrm{ab}}$ & $0,00 \pm 0,00^{\mathrm{a}}$ & $6,16 \pm 0,40^{\mathrm{ab}}$ & $6,32 \pm 0,32^{\mathrm{a}}$ \\
\hline $\mathbf{t 2}$ & $3,74 \pm 0,89^{\mathrm{a}}$ & $0,80 \pm 0,60^{\mathrm{a}}$ & $0,61 \pm 0,55^{\mathrm{a}}$ & $0,08 \pm 0,20^{\mathrm{a}}$ & $1,79 \pm 0,09^{\mathrm{b}}$ & $0,00 \pm 0,00^{\mathrm{a}}$ & $6,21 \pm 0,32^{\mathrm{ab}}$ & $6,16 \pm 0,33^{\mathrm{a}}$ \\
\hline $\mathbf{t 3}$ & $3,69 \pm 0,85^{\mathrm{a}}$ & $0,41 \pm 0,30^{\mathrm{a}}$ & $1,03 \pm 0,20^{\mathrm{a}}$ & $0,12 \pm 0,18^{\mathrm{a}}$ & $1,08 \pm 0,10^{\mathrm{a}}$ & $0,00 \pm 0,00^{\mathrm{a}}$ & $5,97 \pm 0,22^{\mathrm{a}}$ & $6,24 \pm 0,22^{\mathrm{a}}$ \\
\hline
\end{tabular}

Tabla 7. Valor medio en $\log _{10}$ de los parámetros microbiológicos y de RCS. Comparación entre los diferentes tiempos de congelación $(\mathrm{t} 0, \mathrm{t} 1, \mathrm{t} 2$ y t3) $(\mathrm{n}=6)$.

*Medias con letra igual no muestran diferencia significativa $(p<=0,05)$ * RMAT: recuento de mesófilos aerobios totales; CT: coliformes totales; ST: Staphylococcus coagulasa positiva; RCS: recuento de células somáticas.

\begin{tabular}{|c|c|c|c|c|}
\hline \multicolumn{5}{|c|}{ Tiempo inducción (hs) } \\
\hline & $\mathbf{t 0}$ & $\mathbf{t 1}$ & $\mathbf{t} \mathbf{2}$ & $\mathbf{t 3}$ \\
\hline LP H & $31 \pm 8^{\mathrm{a}}$ & $32 \pm 9^{\mathrm{a}}$ & $45 \pm 9^{\mathrm{b}}$ & $45 \pm 8^{\mathrm{b}}$ \\
\hline LP BM & $31 \pm 8^{\mathrm{a}}$ & $31 \pm 9^{\mathrm{a}}$ & $45 \pm 9^{\mathrm{b}}$ & $44 \pm 8^{\mathrm{b}}$ \\
\hline LC H & $32 \pm 10^{\mathrm{a}}$ & $31 \pm 8^{\mathrm{a}}$ & $47 \pm 9^{\mathrm{b}}$ & $44 \pm 9^{\mathrm{b}}$ \\
\hline LC BM & $33 \pm 10^{\mathrm{a}}$ & $31 \pm 11^{\mathrm{a}}$ & $46 \pm 7^{\mathrm{b}}$ & $43 \pm 8^{\mathrm{b}}$ \\
\hline
\end{tabular}

Tabla 8. Valores de estabilidad oxidativa en los diferentes tiempos de congelación (t0, t1, t2 y t3) y en los diferentes tratamientos ( $\mathrm{LPH}$,

LPBM, LCH y LCBM) $(n=6)$.

* Medias con letra igual no muestran diferencia significativa $(p<0,05)$. 


\section{Discusión}

Se evaluó efecto del tratamiento térmico (pasteurización) previo a la congelación en los diferentes parámetros de calidad. El objetivo principal de la pasteurización de la leche es la destrucción de bacterias patógenas, pero también la eliminación de los microorganismos saprófitos y la inactivación de ciertas enzimas que perjudican la calidad del producto (Egito et al., 1989).

La legislación brasileña permite para leche de cabra el uso de pasteurización rápida $\left(72-75{ }^{\circ} \mathrm{C}\right.$ por $\left.15-20 \mathrm{seg}\right)$ o lenta $(62-$ $65{ }^{\circ} \mathrm{C}$ por $30 \mathrm{~min}$ ) (MAPA, 2000). En este trabajo la eficiencia de la pasteurización en la leche de cabra fue comprobada en base a la prueba de fosfatasa alcalina, constatándose siempre su ausencia, así como por la disminución en los recuentos microbiológicos. La ausencia de fosfatasa alcalina ha sido universalmente aceptada como evidencia de una eficiente pasteurización de la leche (Farias et al., 2000). Como puede observarse en la Tabla 4, con la pasteurización de la leche cruda a $63{ }^{\circ} \mathrm{C}$ por 30 minutos se logró disminuir el recuento inicial de mesófilos aerobios totales, el recuento de coliformes totales y la detección de Staphylococcus coagulasa positiva. La apropiada pasteurización de la leche quedó confirmada por la disminución de más de un $99 \%$ del recuento de aerobios mesófilos. Dependiendo de la intensidad del tratamiento térmico, el calentamiento puede provocar cambios en las propiedades fisicoquímicas de la leche, pero estos normalmente son insignificantes cuando la temperatura no excede los $60{ }^{\circ} \mathrm{C}$ (Walstra, 2001). Sin embargo, la leche de cabra tiene menor estabilidad térmica que la bovina (Jenness, 1980), lo que hace que con tratamientos térmicos de la misma intensidad se originen alteraciones diferentes en las leches de ambas especies (Vilhena et al., 2008).

La lactosa está sujeta a alteraciones durante el calentamiento de la leche, principalmente reacciones de Maillard, pero éstas ocurren a temperaturas superiores a $100{ }^{\circ} \mathrm{C}$ (Walstra, 2001), lo cual explica la ausencia de este efecto sobre la lactosa durante la pasteurización.

Al comparar LCBM con LPBM y LCH con LPH se observó que en cuanto a la calidad composicional, al igual que las propiedades fisicoquímicas y recuento de células somáticas, no se encontraron diferencias luego de la congelación (Tablas 2 y 3). Desde el punto de vista microbiológico sí se hallaron diferencias entre los diferentes tratamientos térmicos (Tabla 4), por lo que se deduce que es más adecuado pasteurizar la leche previa a la congelación, dado que se obtiene un producto con una menor carga bacteriana, disminuyendo el riesgo de que se produzcan alteraciones en la composición y propiedades fisicoquímicas causadas por microorganismos (GonzálezRodríguez, 1995).

En relación a los diferentes tratamientos utilizados para la descongelación de la leche, no se detectaron cambios en los parámetros relacionados a la calidad composicional ni a las propiedades fisicoquímicas entre los dos métodos estudiados (Tablas 2 y 3 ), por lo que no habría diferencias, en relación a las características estudiadas, a la hora de decidir por uno u otro método. Vilhena et al. (2008) observaron que utilizando la parte baja de la heladera a temperaturas entre $10-12{ }^{\circ} \mathrm{C}$ lograron un tiempo cercano a las 30 horas para el total descongelado de las muestras. En nuestro trabajo se observó que por el método $\mathrm{H}\left(4^{\circ} \mathrm{C}\right)$ el tiempo de descongelado era de $60 \mathrm{hs}$, mientras que con el método BM el tiempo era de 2,5 hs. Por esto se tiende a pensar que el método $\mathrm{H}$ no sería recomendado para la descongelación de la leche, principalmente a escala industrial, en coincidencia con Vilhena et al. (2008).

A su vez, se evaluó el efecto del tiempo de congelación sobre los parámetros de calidad de la leche caprina cruda (LC) y pasteurizada (LP), ya que diversos autores sostienen que la congelación puede tener efectos adversos en la calidad de la leche (Needs, 1992). No obstante, Benedet y Carvalho (1996) proponen que este proceso de conservación no provoca grandes modificaciones en el sabor ni en el olor de la leche, aunque sí puede ocurrir una floculación de las proteínas, perjudicando la apariencia y aceptación del producto. Al evaluar los parámetros de composición algunos autores afirman que durante la congelación de la leche se producen procesos de lipólisis que provocarían la disminución de los valores de materia grasa (Grappin, 1987). Otros autores sugieren que la disminución de la materia grasa durante la congelación se puede deber a la ruptura enzimática de los triacilglicéridos, junto con la actividad microbiana que se da durante el tiempo de almacenamiento en congelación (González-Rodríguez, 1995). En este trabajo se observó que los valores de materia grasa disminuyeron durante el tiempo de congelado tanto en la LC como en LP. Keenan (2003) afirma que las razones de la reducción del porcentaje de la grasa láctea durante el almacenamiento en congelación no están totalmente comprendidas, aunque es posible que los cristales que se forman durante la congelación puedan destruir los glóbulos de grasa.

En cuanto a las proteínas, Gomes et al. (1997) y Pereira (2000) obtuvieron valores entre $2,70 \%$ y $2,62 \%$ para leche pasteurizada congelada, los cuales coinciden con los encontrados en este estudio. Los agregados de caseína pueden ocurrir en la congelación de la leche, ya que la micela de caseína está fuertemente mineralizada y su grado de hidratación es bajo, lo que le confiere menor estabilidad térmica. La congelación contribuye a disociar la $\beta$-caseína de la micela interfiriendo en la estabilidad proteica. Este efecto ocurre intensamente en la leche de cabra y es probablemente ocasionado por la ausencia de la $\alpha$-S1-caseína (Leach, 1980). Asimismo, la relación calcio-fósforo de la micela es más fuerte y su hidratación es menor, lo cual le da menor estabilidad térmica (Remeuf et al., 1989). La desagregación de la caseína dentro de la micela la hace especialmente susceptible a proteólisis (Fox, 1991). En esta investigación se observa en LC una leve disminución de las proteínas en el transcurso del tiempo, y en LP se registran diferencias a partir de los 120 días en comparación con el control ( $\mathrm{t} 0 \neq \mathrm{t} 2 \mathrm{y}$ t3) (Tabla 5). A pesar de que no se estudió la estabilidad de la micela de caseína, se podría considerar que la disminución en los porcentajes de proteína durante la congelación podría deberse a una menor estabilidad térmica de la proteína al someterla a las temperaturas de congelación $\left(-18^{\circ} \mathrm{C}\right)$. En cuanto a la apariencia de la leche una vez descongelada, Pereira (2000) constató que presentaba un aspecto floculado con disminución de la apariencia general. Lo mismo observaron Gomes et al. (1997), quienes atribuyeron esas características a modificaciones físicas de las proteínas, acentuadas por el congelamiento lento después de la pasteurización. En este estudio no se realizó evaluación sensorial del producto descongelado, pero sí se pudo advertir una declinación en la apariencia general de la leche.

La lactosa, SNG y sales en LC no presentaron diferencias en los distintos tiempos de congelado (Tabla 5). En el caso de la LP, la lactosa presenta una leve disminución a los 120 días, diferencias significativas entre t0 y t2. En referencia a las sales (cenizas), los valores no presentaron diferencias a lo largo de la congelación. Zhang (2006) indica en su trabajo que los sólidos totales, proteínas y porcentajes de lactosa no se vieron afectados por el tiempo de almacenamiento o la temperatura de congelación.

En la Tabla 6 se muestran los parámetros fisicoquímicos para los diferentes tiempos de congelación. El pH en la LC no presentó variación al final del tiempo de congelación. En la LP este parámetro no evidencia diferencias en los primeros 60 días, pero hay diferencia a los 180 días, cuando disminuyó el valor $(\mathrm{t} 0 \neq \mathrm{t} 3)$. Existen controversias al respecto del efecto de la congelación sobre el pH. Así, Dalles et al. (1984) señalaron que permanece inalterado, mientras que Alichanidis et al. (1981) reportaron un aumento del mismo. Durante la congelación, las propiedades fisicoquímicas generales no suelen variar mucho, a excepción de la acidez (Gómez et al., 1998). Guimaraes (1993) y Gomes et al. (1997) indican que la congelación y el almacenamiento de la leche durante una semana y hasta 60 días no alteraba la acidez de la leche. En este trabajo los parámetros de acidez y densidad no presentaron diferencias para LC y LP durante el almacenamiento en congelación en el período de 180 días. Pereira (2000) encontró para la variable densidad valores mínimos y máximos iguales a 1,022 y 1,032, respectivamente, y como media general 1,029 en leche pasteurizada descongelada, lo cual coincide con los valores obtenidos aquí.

En cuanto a los parámetros microbiológicos, Benedet y Schwinden (1991) plantearon que la congelación no altera las 
características microbiológicas de la leche o sus productos y que luego de la descongelación presenta cualidades semejantes a la leche original. La congelación hasta por 90 días de la leche de cabra pasteurizada no altera significativamente sus características microbiológicas. En lo relativo al recuento de mesófilos que se muestra en la Tabla 7, se observó que con el tiempo de congelación sus valores disminuyen en LC. Esta misma tendencia no se mantiene para LP, en la que se evidencia que no existe diferencia en los 180 días que duró el ensayo. Lo que se observó con el RMAT en LC coincide con Le Jaouen (1987), quien indicó que los recuentos de mesófilos disminuyen entre 50 y 100 veces tras cierto tiempo en congelación.

En lo que refiere a los coliformes totales para LC, los recuentos disminuyen, lo cual coincide con la bibliografía referente al tema. En LP los datos muestran (Tabla 7), que no existió diferencia en los recuentos durante los 180 días que se evaluó el efecto de la congelación. Juárez et al. (1987) resaltan la mayor sensibilidad al frío de las bacterias Gram negativas que de las Gram positivas, debido a las diferencias en la estructura de su pared celular. La reducción en la viabilidad de $E$. coli fue demostrada a $-20^{\circ} \mathrm{C}$ por 4 semanas (Pankey et al., 1987). De la misma manera se encontró una reducción en el número de $E$. coli a las 4, 8 y 16 semanas después de congelada (Schukken et al., 1989). En referencia a los estafilococos hallados en leche congelada, Sánchez et al. (2003) reportaron un aumento en el recuento de Staphylococcus coagulasa negativa en los diferentes días de almacenamiento a -20 y $-80{ }^{\circ} \mathrm{C}$. La localización intracelular de los estafilococos y el daño causado a las células fagocitarias en la congelación pueden ser la explicación de este fenómeno (Schukken et al., 1989). Además, y apoyando esta teoría, es conocido que el RCS y el porcentaje de neutrófilos en leche caprina son más altos en comparación con la leche bovina (Paape et al., 1997). En esta investigación no se determinaron los Staphylococcus coagulasa negativa, los cuales siguen una variación similar a Staphylococcus coagulasa positiva, con respecto al cual en el caso de la LC existió variación únicamente entre los 120 y 180 días $(\mathrm{t} 2 \neq \mathrm{t} 3)$. Hasta los 120 días se constató una tendencia creciente en el recuento y luego de 180 días de congelado disminuyó a valores como los obtenidos inicialmente. En la LP no hubo diferencia durante el período congelación, dado que no se encontró este microorganismo en la leche sometida a tratamiento térmico.

En lo vinculado a las células somáticas, no se evidenció aumento en LC ni en LP en los 180 días de congeladas las muestras (Tabla 7). Los investigadores que estudiaron los factores que afectan las células somáticas en leche de cabra (Zeng et al., 1999) indican que el almacenaje de la leche caprina a temperatura de refrigeración $\left(5 \pm 1^{\circ} \mathrm{C}\right)$ por tres días no afecta el recuento, y en relación a la congelación Horner et al. (1988) exponen que tampoco se modifica. Sánchez et al. (2005) encontraron que el rango de variación para el RCS $(p<0.001)$ fue mayor con el almacenaje a temperatura de refrigeración que en la congelación. Por otro lado, en la leche LC se evidencia claramente una disminución del RCS durante los 6 meses que duró la congelación. Esto puede deberse a que durante la misma se forman cristales de hielo que lesionan las células, coincidiendo con Lawrence (1999), quien estudió la congelación en leche humana (cruda) a $-20{ }^{\circ} \mathrm{C}$ y halló una disminución del contenido de células de la línea blanca (macrófagos y linfocitos).

En cuanto a la estabilidad a la oxidación de la leche de cabra en los 180 días de estudio, resulta en un aumento del tiempo de inducción luego de los 4 meses, lo que significa que la leche es más estable a la oxidación tanto a los 4 como a los 6 meses (Tabla 8). En ese sentido, la congelación desde el punto de vista oxidativo de la leche no cambia hasta los 4 meses y mejora luego de este periodo. A propósito de la estabilidad oxidativa, la leche de cabra puede ser congelada cruda o pasteurizada, ya que no presenta diferencia significativa en ambos tratamientos. El descongelamiento de la leche realizado por $\mathrm{H}$ o $\mathrm{BM}$ puede ser utilizado según conveniencia del productor, ya que no se encontraron diferencias estadísticas entre ellas.

\section{Conclusión}

La congelación de la leche caprina durante 6 meses no afectó su calidad en cuanto a los parámetros estudiados, por lo que este método de conservación podría ser una alternativa en establecimientos rurales para mantener un volumen de leche caprina continuo durante todo el año.

No se encontraron diferencias entre los métodos de descongelamiento estudiados, cuya elección se define de acuerdo a la realidad de cada productor $\mathrm{y} / \mathrm{o}$ industria.

Se considera de importancia continuar los estudios en referencia a los efectos de la congelación de la leche utilizada como materia prima para la elaboración de quesos, dado que el $70 \%$ de la leche de cabra producida en el país se destina a la elaboración de estos productos.

\section{Reconocimientos}

Proyecto aprobado y financiado por la Comisión Sectorial de Investigación Científica (CSIC) UdelaR 2009.

Agradecemos al equipo del laboratorio del Departamento de Ciencia y Tecnología de la Leche, Dr. Álvaro González, Lic. Sheila Giacaman y Lic. Karina Cal, por el apoyo en los análisis de laboratorio.

A la Profesora Adjunta Carmen Silvia Gallo Muniz, Encargada del área de inglés de Facultad de Veterinaria de UdelaR, por la corrección en la traducción del resumen al inglés.

A Roberto Silva del Departamento de Cereales, Oleaginosos y Productos Derivados del Laboratorio Tecnológico del Uruguay.

A Laura Olazabal del Departamento de desarrollo de métodos analíticos del Laboratorio Tecnológico del Uruguay.

\section{Referencias}

\section{- ALICHANIDIS, E.; POLYCHRONIADOU, A.; TZANETAKIS, N.;} VAFOPOULOU. Teleme cheese from deep frozen curd. En: Journal of Dairy Sci. 1981, 64:732-739.

- ALUR, M.; GREEZ, N. Mechanism of injury of Escherichia coli by freezing and thawing. En: Biochemical Biophysical Research Communication. 1975, 62:308-312.

- AMERICAN OIL CHEMIST'S SOCIETY (AOCS). Official methods and recommended practices of the American Oil Chemists' Society. 5th ed. Champaign: AOCS, 2009. Official Method Cd 12b-92.

- AMERICAN PUBLIC HEALTH ASSOCIATION (APHA).

Compendium of methods for microbiological examination of food. $4^{\mathrm{a}} \mathrm{ed}$. Washington: APHA, 2001. 676 p.

- ÁVILA, T. Problemática de la leche en México [En línea]. [s.l.]: [s.n.], 2000. [Consulta: 5 de abril de 2009]. Disponible en: http://www.cddhcu. gob.mx/camdip/comlvii/comeco/foro3/m\%E9xico.htm

- BARBERIS, S. Bromatología de la leche. San Luis: Hemisferio Sur, 2002. $228 \mathrm{p}$

- BENEDET, H.; SCHWINDEN, E. Modificaçoes físico-químicas e microbiológicas do leite de cabra, congelado e armazenado. En: Boletim da Sociedade Brasileira de Ciência e Tecnologia de Alimentos. 1991, 25(2):1-76.

- BENEDET, H.; CARVALHO, M. Caracterização do leite de cabra no Estado de Santa Catarina, Brasil. En: Ciência e Tecnologia de Alimentos. 1996, 16(2): 116-119.

- CARDOSO, W. Contagem de microorganismos. En: CARDOSO, W. Analise microbiológica de alimentos. Rio de Janeiro: Merk, 1985. pp. 20-27.

- CIAPPESONI, C. La producción caprina en Uruguay y Latinoamérica [En línea]. [s.1.]: [s.n.], 2006. [Consulta: 28 de setiembre de 2010]. Disponible en: htttp://www.caprahispana.com/mundo/uruguay/uruguay. htm

- DALLES, T.; KALATZOPOULOS, G.; KAEHAGIAS, C. Freezing preservation of soft cheeses with and without mold form goat's and sheep's milk. Thermal prossesing and quality of foods. En: Elsevier Applied Science Publishers. 1984, 91:740-744.

- DE LA FUENTE, M.; REQUENA, T.; JUAREZ, M. Salt balance in ewe's and goat's milk during storage at chilling and freezing 
temperatures. En: Journal of Agricultural and Food Chemistry. 1997, 45(1):82-88.

- EGITO, A. Avaliação da pasteurização lenta do leite de cabra no controle de coliformes totais. En: Boletim de Pesquisa do Centro Nacional de Pesquisa de Caprinos. 1989, 12:12.

- FARIAS, J.; GARCIA, A.; TOVAR, A. Eficacia de la pasteurización de la leche de cabra en una miniplanta procesadora de queso. En: Revista cientifica, FCV-LUZ. 2000, 10(2):119-123.

- FONTECHA, J.; BELLANATO, J.; JUAREZ, M. Infrared and raman spectroscopic study of casein in cheese: effects of freezing and frozen storage. En: Journal of Dairy Science. 1993, 76:3303-3309.

- FOX, P.; LAW, J. Enzymology of cheese ripening. En: Food Biotechnology. 1991, 5(3):239-262.

- GOMES, M.; BONASSI, I.; ROÇA, R. Características químicas, microbiológicas e sensoriais de leite de cabra congelado. En: Ciência e Tecnologia de Alimentos. 1997, 17:111-114.

- GÓMEZ, M.; BONSÁI, A.; ROCA, O. Chemical, microbiological and sensorial characteristics of frozen goat milk. En: Ciência e Tecnologia de alimentos. 1998, 17(2):111-114.

- GONZALEZ-RODRIGUEZ, M.; GONZALO, C.; SAN PRIMITIVO, F.; CARMENES, P. Relationship between somatic cell count and intramammary infection of the half under in dairy cows. En: Journal of Dairy Sci. 1995, 78: 2753-2759.

- GRAPPIN, R. Application of indirect instrumental methods to the measurement of fat and protein content of goat and ewe milk. En: International Dairy Federation Bulletin. 1987, 208:41-43.

- GUIMÃRAES, M. Avaliação da estabilidade físico-quimica de leite caprino congelado durante a estocagem comercial. Belo Horizonte: Escola de Medicina Veterinaria, Universidade Federal de Minas Gerais, 1993. (Tesis de Maestría).

- HAENLEIN, G. Milk and meat products. [En línea]. [s.1.]: [s.n.], [2002] [Consulta: 24 de octubre de 2010]. Disponible en: http://goatconnection. com/articles/publish/article73.shtml

- HORNER, S.; FOX, L. Comparison of somatic cell counting procedures for goat milk. En: Journal of Dairy Science. 1988, 65:275-280.

- INTERNATIONAL DAIRY FEDERATION (Belgium). FIL 148A: Milk enumeration of somatic cells. Brussels: IDF, 1995.

- INTERNATIONAL STANDARD ORGANIZATION (Switzerland). ISO 14156/FIL 172: Milk and milk products. Extraction methods for lipids and liposoluble compounds. Geneve: ISO, 2001.

- JUAREZ, M.; GOICOECHEA, A. Refrigeración y congelación de la leche y productos lácteos. En: Alimentación Equipos y Tecnología.1987, 8(4):133-137.

- KEENAN, T.; MATHER, I. Milk fat globule membrane. En: Encyclopedia of Dairy Sciences. 2003, 3:1568-1576.

- LAWRENCE, R. A. Storage of human milk and the influence of procedures on immunological components of human milk. En: Acta Pediatric. 1999, 88:14-18.

- LEACH, K. Trends in dairy goats. En: Journal Dairy Science. 1980, 63:1600-1604.

- LE JAUOEN, J. La conservation du caillé. En: ECK. A. Le Fromage. Technique et documentation. Paris: Lavoisier, 1987. p. 41-53.

- MESHREF, A.; AL-ROWAILY. effect of heating treatments, processing methods and refrigerated storage of milk and some dairy products on lipids oxidation. En: Pakistan Journal of Nutrition. 2008, 7(1):118125.

- MAPA. MINISTÉRIO DA AGRICULTURA, PECUÁRIA E ABASTECIMENTO. BRASIL. Regulamento técnico de produçao, identidade e qualidade do leite de cabra. Instrução normativa $n^{\circ} 37$, de 31 de octubro de 2000 [En línea]. [Consulta: 26 de julio de 2009]. Disponible en: http://www.agais.com/normas/leite/leitecabra.htm

- MGAP. Manual para habilitación y refrendación de establecimientos productores de leche y queserías artesanales [En línea]. Montevideo: MGAP, 2011. [Consultado el 22 de mayo de 2012]. Disponible en: http://www.mgap.gub.uy/DGSG/DSA/D_ControlLacteos.htm.

- NEEDS, E. Effects of long-term deep-freeze storage on the condition of the fat in raw sheep's milk. En: Journal Dairy Science. 1992, 59:4955.

- PAAPE, M.; CAPUCO, A. Cellular defense mechanisms in the udder and lactation of goats. En: Journal Animal Science. 1997, 75:556-565.

- PANKEY, J.; WADSWORTH, J.; METHA, K.; MURDOUGH, P. Effects of storage on viability of mastitis pathogens. En: Journal Dairy Science. 1987, 70 (suppl.1):132.

- PEREIRA, V. Avaliação da qualidade microbiológica e características físico-químicas do leite de cabra pasteurizado, congelado, comercializado na região Centro-Oeste do Estado de São Paulo. San Pablo: Faculdade de Medicina Veterinária e Zootecnia, Universidade Estadual Paulista, 2000

- PINTO, M.; VEGA, S.; LEON, S. Métodos de análisis de la leche y derivados. Valdivia: Ediciones Universidad Austral de Chile, 1998. 489 $\mathrm{p}$.

- REMEUF, F.; LENOIR, T.; DUBY, C. Étude des relations entre les caractéristiques physicochimiques des laits de chèvre et leur aptitude à la coagulation par la présure. En: Lait. 1989, 69:499-518.

- SÁNCHEZ, A.; CONTRERAS, A.; JIMÉNEZ, J.; LUENGO, C.; CORRALES, J.; FERNÁNDEZ, C. Effect of freezing goat milk samples on recovery of intramammary bacterial pathogens. En: Veterinary Microbiology. 2003, 94:71-77.

- SÁNCHEZ, A.; SIERRA, D.; LUENGO, C.; CORRALES, J.; MORALES, C.; CONTRERAS, A.; GONZALO, C. Influence of storage and preservation on fossomatic cell count and composition of goat milk. En: Journal Dairy Science. 2005, 88:3095-3100.

- SCHUKKEN, Y.; SMIT, J.; GROMMERS, F.; VANDEGEER, D.; BRAND, A. Effect of freezing on bacteriologic culturing of mastitis milk samples. En: Journal Dairy Science. 1989, 72:1900-1906.

- SILVA, P.; PEREIRA, D.; OLIVEIRA, L.; COSTA JUNIOR, L. FísicoQuímica do leite e derivados. Minas Gerais: Oficina de Impressão Gráfica e Editora Ltda. Juiz de Fora, 1997.

- VILHENA, P.; RESENDE DE SOUZA, M.; FREIRE, C.; FERREIRA, J. Características microbiológicas e físico-químicas do leite de cabra submetido à pasteurizaçao lenta pós-envase e ao congelamento. En: Ciencia Rural. 2008, 38(5):1424-1430.

- VELASCO, J.; ANDERSEN, M.L.; SKIBSTED, L.H. Evaluation of oxidative stability of vegetable oils by monitoring the tendency to radical formation. A comparison of electron spin resonance spectroscopy with the Rancimat method and differential scanning calorimetry. En: Food Chemistry. 2004, 85:623-632.

- WALSTRA, P. Ciencia de la leche y tecnología de los productos lácteos. Madrid: Acribia, 2001. ISBN: 842000961X.

- ZENG, S.; ESCOBAR, E.; HART, S.; HINCKLEY, L.; BAULTHAUS, M.; ROBINSON, T.; JAHNKE, G. Comparative study of the effects of testing laboratory, counting method, storage and shipment on somatic cell count in goat milk. En: Small Ruminant Reserch. 1999, 31:103107.

- ZHANG, R.; MUSTAFA, A.; NG-KWAI-HANG, K.; ZHAO X. Effects of freezing on composition and fatty acid profiles of sheep milk and cheese. En: Small Ruminant Research. 2006, 64:203-210. 